\title{
Effects of the Characteristics and Duration of Chronic Pain on Psychosomatic Function in the Community-Dwelling Elderly Population
}

\author{
Mitsumasa Hida $\mathbb{D}^{1},{ }^{1,2,3}$ Misa Nakamura $\mathbb{D}^{1},{ }^{1,2}$ Masakazu Imaoka, ${ }^{1,2}$ Hidetoshi Nakao, ${ }^{1,2}$ \\ Fumie Tasaki, ${ }^{1,2}$ Tomoko Omizu, ${ }^{1,2}$ Masatoshi Takeda, ${ }^{1,2}$ Tadasuke Ohnishi, ${ }^{4}$ \\ and Chikamune Wada ${ }^{3}$ \\ ${ }^{1}$ Department of Rehabilitation, Osaka Kawasaki Rehabilitation University, Mizuma 158, Kaizuka, Osaka 597-0104, Japan \\ ${ }^{2}$ Cognitive Reserve Research Center, Osaka Kawasaki Rehabilitation University, Mizuma 158, Kaizuka, Osaka 597-0104, Japan \\ ${ }^{3}$ Graduate School of Life Science and Systems Engineering, Kyushu Institute of Technology, 2-4 Hibikino, Wakamatsu-ku, \\ Kitakyushu 808-0196, Japan \\ ${ }^{4}$ Frontier Community Rehabilitation Center, Showa-Inan General Hospital, Akaho 3230, Komagane, Nagano 399-4117, Japan
}

Correspondence should be addressed to Mitsumasa Hida; hidam@kawasakigakuen.ac.jp

Received 1 November 2019; Accepted 18 March 2020; Published 7 April 2020

Academic Editor: Giustino Varrassi

Copyright (c) 2020 Mitsumasa Hida et al. This is an open access article distributed under the Creative Commons Attribution License, which permits unrestricted use, distribution, and reproduction in any medium, provided the original work is properly cited.

\begin{abstract}
Catastrophic thinking is related to pain intensity and the degree of disability and influences pain care significantly. However, only few studies have investigated the impact of catastrophic thinking on chronic pain (CP) in the community-dwelling elderly population. This study aimed to evaluate the characteristics of $\mathrm{CP}$ in the community-dwelling elderly population and to investigate the effects of different periods of CP on cognitive and psychological functions. A total of 187 community-dwelling elderly people met the inclusion criteria and were included in this cross-sectional study. The survey items included demographic data (age and gender), pain-related questionnaires, psychological and cognitive functions, and sleep status. The duration of CP was investigated using three categories: no pain and pain for $\leq 1$ year and $\geq 1$ year. A logistic regression analysis was performed to identify the factors most strongly associated with the presence of CP. The difference in each assessment was compared according to duration of $\mathrm{CP}$ among the three groups and analyzed using the chi-square test, Kruskal-Wallis test, and one-way analysis of variance. The PCS scores and depression scores were significantly higher in long duration of CP compared with no pain and pain for $\leq 1$ year. The present study is consistent with the fear-avoidance model and was concluded that community-dwelling elderly people with CP are depressive and tend to magnify their pain with long duration of $\mathrm{CP}$.
\end{abstract}

\section{Introduction}

Chronic pain (CP), defined as "pain that extends beyond the expected period of healing or progressive pain due to noncancer diseases," generally lasts for more than 12 weeks $[1,2]$. It is reported to be affected by genetic, environmental, and cultural factors, in addition to socioeconomic status and psychological factors [3]. This suggests that $\mathrm{CP}$ is caused by a variety of experiences that affect the individual, regardless of age.
CP is present in $20 \%-25 \%$ of the total population and is related to decline in the quality of life (QOL) and physical functions [4]. Nakamura et al. revealed that CP is associated with a decrease in all domains of the 36-Item Short Form Survey (SF-36) questionnaire QOL score [5]. Additionally, $10 \%$ of patients with chronic musculoskeletal pain are limited from attending school or work and require longterm treatment, thereby significantly impacting the medical economics [5]. In Japan, rapid aging of the population has increased the number of community-dwelling elderly people 
who require long-term care due to decline in psychosomatic functions $[6,7]$. Previous studies have shown that $\mathrm{CP}$ is associated with increased isolation and fatigue and decreased subjective health, $\mathrm{ADL}$, and motor functions in communitydwelling elderly people [8-10].

Cheng et al. revealed that catastrophic thinking mediates the relationship between pain intensity and depressive symptoms and self-efficacy mediates the relationship between pain intensity and catastrophic thinking [11]. Catastrophic thinking of pain was proposed by Ellis [12] and is defined as "an exaggerated negative mental set brought to bear during actual or anticipated painful experience"; it is a typical cognitive factor for CP [13]. It has been indicated that catastrophic thinking is related to the pain intensity and the degree of disability and has an important influence on the care of pain $[14,15]$. However, only few studies have investigated the impact of catastrophic thinking on $\mathrm{CP}$ in the community-dwelling elderly population $[16,17]$. Hirase et al. revealed that the factor most strongly associated with musculoskeletal CP in community-dwelling elderly people who exercise regularly is the helplessness subscale of the Pain Catastrophizing Scale (PCS) [16]. However, there are individuals who do not exercise regularly in the communitydwelling elderly people; therefore, it is necessary to investigate the general community-dwelling elderly people. Breivik et al. conducted a survey comprising 4839 subjects with CP in Europe; a total of $40 \%$ of those with CP did not visit the hospital and only $2 \%$ were treated by pain management specialists. One-third of them revealed that they were not currently receiving treatment [18]. In Japan, reports suggest that only $42 \%$ of patients with $\mathrm{CP}$ receive folk remedies and treatment at medical facilities [5]. The community-dwelling elderly who remain under sufficient care may have negative health-economic impacts as they progress to $\mathrm{CP}$ and visit the healthcare facility only after the symptoms worsen. Investigating the cognitive and psychological characteristics, including catastrophic thinking, in individuals with long CP is important for the planning of appropriate prevention and early intervention. Therefore, this study aims to evaluate the characteristics of $\mathrm{CP}$ in the community-dwelling elderly and to investigate the effects of different periods of $\mathrm{CP}$ on cognitive and psychological functions.

\section{Materials and Methods}

2.1. Subjects. This cross-sectional study was approved by the Osaka Kawasaki Rehabilitation University Research Ethics Review Board (approval number: OKRU-A016), and written informed consent was obtained from each participant prior to study inclusion. A total of 215 community-dwelling elderly people who underwent health checks in Kaizuka City, Osaka, from August to September 2018 participated in this study. Participant inclusion criteria were as follows: aged $\geq 65$ years and living at home. Individuals unable to respond to interview questions because of a cognitive impairment were excluded. Of the 215 potential participants, 21 (9.8\%) were excluded because their age was under 65 years and 7 (3.3\%) were excluded because the data provided were incomplete; therefore, 187 participants $(87.0 \%)$ met the inclusion criteria and were included in the study (average age, $74.7 \pm 6.0$ years; males, 47 ; females, 140 ). The presence or absence of CP was ascertained in accordance with the following definition of $\mathrm{CP}$ : associated with a lesion that persists or recurs beyond 3 months or persists beyond 1 month after recovery from acute tissue damage or does not cure [2]. The subjects were required to indicate the presence or absence of pain by responding to "Yes" and "No" questions in a self-reported questionnaire; those who answered "Yes" were enrolled in the study.

2.2. Methods. The survey items included in this study were demographic data (age and gender), pain-related surveys, psychological functions, cognitive functions, and sleep status. The duration of $\mathrm{CP}$ was investigated using three categories: no pain, pain for less than 1 year, and pain for more than 1 year. The PCS, a 13-item self-reported measure comprising rumination, magnification, and helplessness, was used in this study [13]. The psychological function assessment was based on the Geriatric Depression Scale 15 (GDS-15) [19]. It is a test consisting of 15 items with "Yes" or "No" answers. Each item is calculated as 1 point with 15 points in total; the higher the score, the more severe the depression. Cognitive functions were investigated using the Mini-Mental State Examination, which consisted of items such as orientation of time and place, memorizing ability, calculation, recall ability, sentence creation, and graphic replication [20]. It is evaluated using a 30-point scale wherein the lower the score, the lower the cognitive function. The sleep state was investigated using the Japanese version of the Pittsburgh Sleep Quality Index (PSQI-J), a questionnaire composed of 18 items about sleep quality, which was developed by Buysse et al. [21, 22]. The questionnaire consists of questions about sleep habits and sleep quality over the past month with a cutoff value of six points. The PSQI is reported to have good validity and to be highly reliable. Additionally, the number of falls over the past year was investigated.

2.3. Statistical Analysis. The Shapiro-Wilk test was used to confirm the normality of the distribution of each evaluation item. The endpoints in the $\mathrm{CP}$ and non-CP (NCP) groups were compared using the chi-square test, Mann-Whitney's $U$ test, and unpaired $t$-test. A logistic regression analysis was performed to identify the factors most strongly associated with the presence of CP. Variance inflation factors for independent variables were calculated to assess multicollinearity between the independent variables. A variance inflation factor $>10$ was considered to show multicollinearity. Comparison of about the difference in each evaluation item according to duration of $\mathrm{CP}$ was compared among the three groups and analyzed using the chi-square test, Kruskal-Wallis test with Scheffe comparison, and oneway analysis of variance with Bonferroni comparison. The statistical software used was IBM SPSS Statistics 26 (IBM Corp., Armonk, NY, US) with a significance level of less than $5 \%$. 
TABLE 1: Comparison between groups based on chronic pain.

\begin{tabular}{lcccc}
\hline & & NCP & CP & Total \\
\hline Subject & & 102 & 85 & 187 \\
Age (year) & Male & $74.7 \pm 6.0$ & $74.4 \pm 5.9$ & $74.7 \pm 6.0$ \\
Sex & Rumination & $28(27.5 \%)$ & $19(22.4 \%)$ & $47(25.1 \%)$ \\
& Magnification & $5.2 \pm 5.8$ & $10.4 \pm 5.9^{* *}$ & $7.6 \pm 6.4$ \\
PCS (points) & Helplessness & $1.9 \pm 2.8$ & $4.2 \pm 3.5^{* *}$ & $3.0 \pm 3.3$ \\
& Total score & $2.6 \pm 3.4$ & $6.0 \pm 5.2^{* *}$ & $4.1 \pm 4.6$ \\
GDS (points) & & $9.7 \pm 10.9$ & $20.5 \pm 13.4^{* *}$ & $3.8 \pm 2.8^{*}$ \\
Number of falls (frequency) & & $2.8 \pm 2.2$ & $1.2 \pm 3.1^{* *}$ & $3.3 \pm 2.5$ \\
PSQI (points) & & $0.3 \pm 0.8$ & $5.7 \pm 4.0$ & $0.7 \pm 2.2$ \\
MMSE (points) & $5.1 \pm 3.2$ & $28.2 \pm 2.3$ & $5.4 \pm 3.6$ \\
\hline
\end{tabular}

Mean \pm SD, ${ }^{*} p<0.05$, and ${ }^{* *} p<0.01$. CP: chronic pain, NCP: nonchronic pain, PCS: Pain Catastrophizing Scale, GDS: Geriatric Depression Scale, PSQI: Pittsburgh Sleep Quality Index, and MMSE: Mini-Mental State Examination.

\section{Results}

Table 1 shows the results of the univariate analysis in the $\mathrm{CP}$ and NCP groups. The total PCS score, rumination, helplessness, magnification, GDS, and number of falls were significantly different between the two groups. The results of the logistic regression analysis are shown in Table 2. Analyses were conducted with $\mathrm{CP}$ presence as the dependent variable. Items demonstrating significant differences in previous between-group comparisons were designated as the independent variables. Variance inflation factors for all independent variables were $<4$, indicating no multicollinearity between the variables. Based on this analysis, PCS rumination score (odds ratio $=1.11$ ) and number of falls (odds ratio $=1.37$ ) were identified as significant factors associated with the presence of $\mathrm{CP}$.

The NCP group comprised 102 patients, the group with pain for less than 1 year comprised 27 patients, and the group with pain for more than 1 year consisted of 58 patients (Table 3). The items that appeared to exert the main effects were total PCS score, rumination, helplessness, magnification, and PSQI-J. The total PCS scores, magnification, and helplessness in the two groups with CP (less than and more than 1 year) were significantly different from those in the NCP group. In addition, GDS was significantly higher in the group with $\mathrm{CP}$ for more than 1 year compared with the NCP group. Furthermore, in the group with CP for $\geq 1$ year, GDS and PCS magnification was significantly higher than that in in the group with $\mathrm{CP}$ for $\leq 1$ year.

\section{Discussion}

Vlaeyen and Linton advocated the fear-avoidance model as a vicious circle of pain according to which pain causes catastrophe by negatively impacting the thoughts, emotions, cognition, and behavior; this gradually leads to the development of $\mathrm{CP}$ as a result of inactivity, depression, and reduced ability, thereby worsening the symptoms [23]. The results of the present study support the fear-avoidance model. Individuals with CP had higher PCS scores than those with NCP, leading to progressive catastrophic pain. Furthermore, the scales for evaluating depressive symptoms and number of falls were also significantly higher among
TABLE 2: Multiple logistic regression analysis for odds ratio and 95\% confidence interval of chronic pain.

\begin{tabular}{|c|c|c|c|}
\hline & $\begin{array}{l}\text { Odds } \\
\text { ratio }\end{array}$ & $\begin{array}{l}95 \% \text { confidential } \\
\text { interval }\end{array}$ & $\begin{array}{c}P \\
\text { value }\end{array}$ \\
\hline Rumination & 1.11 & $1.01-1.21$ & 0.02 \\
\hline Magnification & 0.97 & $0.80-1.16$ & 0.97 \\
\hline Helplessness & 1.10 & $0.97-1.24$ & 0.14 \\
\hline Number of falls & 1.37 & $1.01-1.86$ & 0.04 \\
\hline GDS & 1.07 & $0.92-1.23$ & 0.38 \\
\hline
\end{tabular}

Data were adjusted by age and sex. PCS: Pain Catastrophizing Scale and GDS: Geriatric Depression Scale.

those with CP. PCS rumination was found to be related to $\mathrm{CP}$ in the elderly living in the region. Rumination refers to the tendency to repeatedly think about the pain [24]. Previous studies suggest that the subitems of PCS are strongly associated with pain intensity and interference $[25,26]$. Pain interference refers to the extent to which pain limits or interferes with an individual's physical, mental, and social activities. Our results support the study by Adachi et al., wherein Japanese individuals tended to concentrate on pain sensation, thus, having a negative impact on daily life [26].

The vicious circle of the fear-avoidance model is presumed to be accompanied by a certain period. Therefore, the disability could be worsened when the duration of $\mathrm{CP}$ is prolonged. The findings of the current study indicate that early intervention is required for $\mathrm{CP}$ in the communitydwelling elderly. First, all the subscales of the PCS were higher in patients with $\mathrm{CP}$ compared with those in the NCP group; the scores were higher as the duration of $\mathrm{CP}$ increased. Second, GDS, which evaluates depressive symptoms, was significantly higher in the group with $\mathrm{CP}$ for over 1 year compared with the group with NCP. It is well known that catastrophic thinking has a significant effect on depression, stress, functional ability, and disability [27, 28]. In addition, the results of this study revealed that all of the PCS subscale scores were higher and the depression scores were significantly higher when $\mathrm{CP}$ persisted for more than one year. Therefore, community-dwelling elderly people with $\mathrm{CP}$ must receive appropriate care within a year. The group with $\mathrm{CP}$ for more than 1 year had significantly higher PCS magnification and GDS scores than those with CP for less than 1 year. Magnification refers to the tendency to magnify 
TABLE 3: Comparisons between nonchronic pain, chronic pain (less than 1 year), and chronic pain (more than 1 year) groups.

\begin{tabular}{|c|c|c|c|c|c|c|c|}
\hline & & \multirow{2}{*}{$\operatorname{NCP}(N=102)$} & \multicolumn{2}{|c|}{$\mathrm{CP}$} & \multirow{2}{*}{ NCP vs. CP ( $\leq 1$ year) } & \multirow{2}{*}{ NCP vs. CP ( $\geq 1$ year $)$} & \multirow{2}{*}{$\begin{array}{c}\mathrm{CP}(\leq 1 \text { year }) \text { vs. } \\
\mathrm{CP}(\geq 1 \text { year })\end{array}$} \\
\hline & & & $\leq 1$ year $(N=27)$ & $\geq 1$ year $(N=58)$ & & & \\
\hline \multirow{2}{*}{$\begin{array}{l}\text { Age }^{a} \\
\operatorname{Sex}^{c}\end{array}$} & & $74.7 \pm 6.0$ & $73.8 \pm 5.8$ & $75.1 \pm 6.0$ & Ns & Ns & Ns \\
\hline & Male & $28(27.5 \%)$ & $4(14.8 \%)$ & $15(25.9 \%)$ & Ns & Ns & Ns \\
\hline \multirow{4}{*}{$\mathrm{PCS}^{b}$} & Rumination & $5.2 \pm 5.8$ & $8.9 \pm 5.6$ & $11.1 \pm 5.9$ & $<0.01$ & $<0.01$ & Ns \\
\hline & Magnification & $1.9 \pm 2.8$ & $2.6 \pm 2.8$ & $4.9 \pm 3.6$ & Ns & $<0.01$ & $<0.01$ \\
\hline & Helplessness & $2.6 \pm 3.4$ & $5.0 \pm 5.0$ & $6.4 \pm 5.3$ & $<0.05$ & $<0.01$ & Ns \\
\hline & Total & $9.7 \pm 10.9$ & $16.4 \pm 12.0$ & $22.4 \pm 13.7$ & $<0.05$ & $<0.01$ & Ns \\
\hline \multicolumn{2}{|c|}{$\mathrm{GDS}^{b}$} & $2.8 \pm 2.2$ & $2.7 \pm 1.7$ & $4.3 \pm 3.0$ & Ns & $<0.05$ & $<0.01$ \\
\hline \multicolumn{2}{|c|}{ Number of falls ${ }^{b}$} & $0.3 \pm 0.8$ & $0.5 \pm 0.9$ & $1.5 \pm 3.7$ & Ns & $<0.01$ & Ns \\
\hline \multicolumn{2}{|c|}{$\mathrm{PSQI}^{b}$} & $5.1 \pm 3.2$ & $5.0 \pm 4.0$ & $6.1 \pm 4.0$ & Ns & Ns & Ns \\
\hline \multicolumn{2}{|c|}{$\operatorname{MMSE}^{b}$} & $27.9 \pm 2.8$ & $28.1 \pm 2.7$ & $28.3 \pm 2.1$ & Ns & Ns & Ns \\
\hline
\end{tabular}

${ }^{a}$ One-way ANOVA (multiple comparison: Bonferroni); ${ }^{b}$ Kruskal-Wallis test (multiple comparison: Scheffe); ${ }^{c}$ chi-square test. CP, chronic pain; NCP, nonchronic pain; PCS, Pain Catastrophizing Scale; GDS, Geriatric Depression Scale; PSQI, Pittsburgh Sleep Quality Index; MMSE, Mini-Mental State Examination.

the intensity of pain and the problems caused by it [4]. Rodero et al. investigated the relationship between catastrophic thinking and pain duration in patients with $<2$ years, 2-4 years, and $>4$ years of fibromyalgia and found that rumination was a significant predictor in the $<2$ year group, magnification and helplessness in the period of 2-4 years group, and helplessness in the $>4$ years group [29]. In other words, differences in the duration of $\mathrm{CP}$ can affect the subscale of catastrophic thinking. Our findings did not agree with those reported by Rodero et al. because the subjects in the current study comprised community-dwelling elderly people. However, initially our study population did present with significantly higher values for all the PCS items. When the duration of pain exceeded 1 year, the number of magnified items and the intensity of pain increased, suggesting the presence of mental disorders such as depression.

The limitation of this study is that it has not investigated the characteristics of subjects with $\mathrm{CP}$ for more than a year. It has been reported that the morbidity of knee osteoarthritis, lumbar spondylosis, and osteoporosis, which are cited as one of the causes of CP, increases with age [30]. Therefore, the subjects of this study who were assigned to groups with $\mathrm{CP}$ of more than 1 year may have experienced $\mathrm{CP}$ for a longer period. In addition, subjects who have $\mathrm{CP}$ for a longer period may not have been able to participate in this study due to a decline in mobility. Additional surveys including these patients need to be conducted.

In this study, we found that Japanese patients with $\mathrm{CP}$ have a high degree of resistance to pain, and as the period of $\mathrm{CP}$ increases, the tendency to magnify the pain increases. This exerts an adverse effect on daily activities and social functions. Rehabilitation with activities such as physical activity, exercise therapy, and cognitive behavior therapy is important for the treatment of CP [31]. These can be implemented by local residents; hence, it is important to raise the awareness of health workers in the community.

\section{Conclusions}

We evaluated the characteristics of $\mathrm{CP}$ in the communitydwelling elderly and investigated the effects of different periods of $\mathrm{CP}$ on cognitive and psychological functions. PCS rumination score and number of falls were identified as significant factors associated with the presence of CP. Additionally, individuals with longer $\mathrm{CP}$ were suggested to have a worse PCS rumination score and GDS. Early care must be provided to community-dwelling elderly population with CP.

\section{Data Availability}

The data used to support the findings of this study are available from the corresponding author upon request.

\section{Conflicts of Interest}

The authors declare no conflicts of interest.

\section{References}

[1] H. Merskey and N. Bogduk, IASP Task Force on Taxonomy, Classification of Chronic Pain, pp. 209-214, IASP Press, Seattle, WC, USA, 2nd edition, 1994.

[2] J. I. Bonica, "General consideration of chronic pain," The Management of Pain, pp. 180-196, Bonica JI, Lea \& Febiger, Philadelphia, PA, USA, 2nd edition, 1990.

[3] P. Brooks, "Issues with chronic musculoskeletal pain," Rheumatology, vol. 44, no. 7, pp. 831-833, 2005.

[4] M. M. Ohayon and A. F. Schatzberg, "Using chronic pain to predict depressive morbidity in the general population," Archives of General Psychiatry, vol. 60, no. 1, pp. 39-47, 2003.

[5] M. Nakamura, Y. Toyama, Y. Nishiwaki, and T. Ushida, "Prevalence and characteristics of chronic musculoskeletal pain in Japan," Journal of Orthopaedic Science, vol. 16, no. 4, pp. 424-432, 2011.

[6] S. Inokuchi, N. Matsusaka, T. Hayashi, and H. Shindo, "Feasibility and effectiveness of a nurse-led community exercise programme for prevention of falls among frail elderly people: a multi-centre controlled trial," Journal of Rehabilitation Medicine, vol. 39, no. 6, pp. 479-485, 2007.

[7] H. Arai, Y. Ouchi, K. Toba et al., "Japan as the front-runner of super-aged societies: perspectives from medicine and medical care in Japan," Geriatrics \& Gerontology International, vol. 15, no. 6, pp. 673-687, 2015.

[8] S. Rapo-Pylkkö, M. Haanpää, and H. Liira, "Chronic pain among community-dwelling elderly: a population-based 
clinical study," Scandinavian Journal of Primary Health Care, vol. 34, no. 2, pp. 159-164, 2016.

[9] Y. Nakai, H. Makizako, R. Kiyama et al., "Association between chronic pain and physical frailty in community-dwelling older adults," International Journal of Environmental Research and Public Health, vol. 16, no. 8, p. 1330, 2019.

[10] T. Hirase, H. Kataoka, J. Nakano, S. Inokuchi, J. Sakamoto, and M. Okita, "Impact of frailty on chronic pain, activities of daily living and physical activity in community-dwelling older asdults: a cross-sectional study," Geriatrics \& Gerontology International, vol. 18, no. 7, pp. 1079-1084, 2018.

[11] S. T. Cheng, C. M. C. Leung, K. L. Chan et al., "The relationship of self-efficacy to catastrophizing and depressive symptoms in community-dwelling older adults with chronic pain: a moderated mediation model," PLoS One, vol. 13, no. 9, Article ID e0203964, 2018.

[12] A. Ellis, Reason and Emotion in Psychotherapy, Lyle-Stuart, New York, NY, USA, 1962.

[13] M. J. L. Sullivan, S. R. Bishop, and J. Pivik, "The pain catastrophizing scale: development and validation," Psychological Assessment, vol. 7, no. 4, pp. 524-532, 1995.

[14] M. J. Sorbi, M. L. Peters, D. A. Kruise et al., "Electronic momentary assessment in chronic pain I: psychological pain responses as predictors of pain intensity," The Clinical Journal of Pain, vol. 22, no. 1, pp. 55-66, 2006.

[15] M. J. Sorbi, M. L. Peters, D. A. Kruise et al., "Electronic momentary assessment in chronic pain II: pain and psychological pain responses as predictors of pain disability," The Clinical Journal of Pain, vol. 22, no. 1, pp. 67-81, 2006.

[16] T. Hirase, H. Kataoka, S. Inokuchi, J. Nakano, J. Sakamoto, and M. Okita, "Factors associated with chronic musculoskeletal pain in Japanese community-dwelling older adults: a cross-sectional study," Medicine (Baltimore), vol. 96, no. 23, Article ID e7069, 2017.

[17] R. Severeijns, M. A. van den Hout, J. W. Vlaeyen, and S. J. H. Picavet, "Pain catastrophizing and general health status in a large Dutch community sample," Pain, vol. 99, no. 1-2, pp. 367-376, 2002.

[18] H. Breivik, B. Collett, V. Ventafridda, R. Cohen, and D. Gallacher, "Survey of chronic pain in Europe: prevalence, impact on daily life, and treatment," European Journal of Pain, vol. 10, no. 4, p. $287,2006$.

[19] K. Nakamura and T. Ogata, "Locomotive syndrome: definition and management," Clinical Reviews in Bone and Mineral Metabolism, vol. 14, no. 2, pp. 56-67, 2016.

[20] E. Oudman, A. Postma, S. Van der Stigchel, B. Appelhof, J. W. Wijnia, and T. C. W. Nijboer, "The montreal cognitive assessment (MoCA) is superior to the mini mental state examination (MMSE) in detection of korsakoff's syndrome," The Clinical Neuropsychologist, vol. 28, no. 7, pp. 1123-1132, 2014.

[21] D. J. Buysse, C. F. Reynolds 3rd., T. H. Monk, S. R. Berman, and D. J. Kupfer, "The Pittsburgh sleep quality index: a new instrument for psychiatric practice and research," Psychiatry Research, vol. 28, no. 2, pp. 193-213, 1989.

[22] Y. Doi, M. Minowa, M. Uchiyama et al., "Psychometric assessment of subjective sleep quality using the Japanese version of the pittsburgh sleep quality index (PSQI-J) in psychiatric disordered and control subjects," Psychiatry Research, vol. 97, no. 2-3, pp. 165-172, 2000.

[23] J. W. S. Vlaeyen and S. J. Linton, "Fear-avoidance and its consequences in chronic musculoskeletal pain: a state of the art," Pain, vol. 85, no. 3, pp. 317-332, 2000.
[24] M. Sullivan, The Pain Catastrophizing Scale: User Manual Montreal, pp. 1-36, Mcgill University, Montreal, Canada, 2009.

[25] A. Osman, F. X. Barrios, B. A. Kopper, W. Hauptmann, J. Jones, and E. O'Neill, "Factor structure, reliability, and validity of the pain catastrophizing scale," Journal of Behavioral Medicine, vol. 20, no. 6, pp. 589-605, 1997.

[26] T. Adachi, A. Nakae, T. Maruo et al., "The relationships between pain-catastrophizing subcomponents and multiple pain-related outcomes in Japanese outpatients with chronic pain: a cross-sectional study," Pain Practice, vol. 19, no. 1, pp. 27-36, 2019.

[27] J. Briest and M. Bethge, "Der einfluss von katastrophisieren auf den effekt von depressivität auf schmerz und körperliche funktion," Der Schmerz, vol. 31, no. 2, pp. 159-166, 2017.

[28] R. Severeijns, J. W. S. Vlaeyen, M. A. van den Hout, and W. E. J. Weber, "Pain catastrophizing predicts pain intensity, disability, and psychological distress independent of the level of physical impairment," The Clinical Journal of Pain, vol. 17, no. 2, pp. 165-172, 2001.

[29] B. Rodero, B. Casanueva, J. García-Campayo, M. Roca, R. Magallón, and Y. L. del Hoyo, "Stages of chronicity in fibromyalgia and pain catastrophising: a cross-sectional study," BMC Musculoskelet Disord, vol. 11, no. 1, p. 251, 2010.

[30] N. Yoshimura, S. Muraki, H. Oka et al., "Prevalence of knee osteoarthritis, lumbar spondylosis, and osteoporosis in Japanese men and women: the research on osteoarthritis/osteoporosis against disability study," Journal of Bone and Mineral Metabolism, vol. 27, no. 5, pp. 620-628, 2009.

[31] R. J. Gatchel, D. D. McGeary, C. A. McGeary, and B. Lippe, "Interdisciplinary chronic pain management: past, present, and future," American Psychologist, vol. 69, no. 2, pp. 119-130, 2014. 